Bull. Soc. math. France

134 (3), 2006, p. 383-394

\title{
A CLASS OF NON-RATIONAL SURFACE SINGULARITIES WITH BIJECTIVE NASH MAP
}

\author{
By Camille Plénat \& Patrick Popescu-Pampu
}

\begin{abstract}
Let $(\mathcal{S}, 0)$ be a germ of complex analytic normal surface. On its minimal resolution, we consider the reduced exceptional divisor $E$ and its irreducible components $E_{i}, i \in I$. The Nash map associates to each irreducible component $C_{k}$ of the space of arcs through 0 on $\mathcal{S}$ the unique component of $E$ cut by the strict transform of the generic arc in $C_{k}$. Nash proved its injectivity and asked if it was bijective. As a particular case of our main theorem, we prove that this is the case if $E \cdot E_{i}<0$ for any $i \in I$.

RÉSUMÉ (Une classe de singularités non-rationnelles de surfaces ayant une application de Nash bijective)

Soit $(\mathcal{S}, 0)$ un germe de surface analytique complexe normale. Nous considérons le diviseur exceptionnel réduit $E$ et ses composantes irréductibles $E_{i}, i \in I$ sur sa résolution minimale. L'application de Nash associe à chaque composante irréductible $C_{k}$ de l'espace des arcs passant par 0 sur $\mathcal{S}$, l'unique composante de $E$ rencontrée par la transformée stricte de l'arc générique dans $C_{k}$. Nash a prouvé son injectivité et a demandé si elle était bijective. Nous prouvons que c'est le cas si $E \cdot E_{i}<0$ pour tout $i \in I$ comme cas particulier de notre théorème principal.
\end{abstract}

Texte reçu le 21 janvier 2005, révisé le 24 mai 2005.

Camille Plénat, Université de Provence, LATP UMR 6632, Centre de Mathématiques et Informatique, 39 rue Joliot-Curie, 13453 Marseille Cedex 13 (France).

E-mail : plenat@cmi.univ-mrs.fr

Patrick Popescu-Pampu, Université Paris 7 Denis Diderot, Institut de Mathématiques, UMR CNRS 7586, Équipe Géométrie et Dynamique, case 7012, 2, place Jussieu, 75251 Paris Cedex 05 (France). • E-mail : ppopescu@math.jussieu.fr

2000 Mathematics Subject Classification. - 14B05, 32S25, 32S45.

Key words and phrases. - Space of arcs, Nash map, Nash problem. 


\section{Introduction}

Let $(\mathcal{S}, 0)$ be a germ of complex analytic normal surface. Let

$$
\pi_{m}:\left(\widetilde{\mathcal{S}}_{m}, E\right) \longrightarrow(\mathcal{S}, 0)
$$

be its minimal resolution, where $E$ is the reduced exceptional divisor of $\pi_{m}$, and let $\left(E_{i}\right)_{i \in I}$ be the irreducible components of $E$. A resolution is called good if $E$ has normal crossings and if all its components are smooth. It is important to notice that, by Grauert's Contractibility Theorem for surfaces [5], there exist singularities whose minimal resolution is not good.

An arc through 0 on $\mathcal{S}$ is a germ of formal map $(\mathbb{C}, 0) \rightarrow(\mathcal{S}, 0)$.

We denote by $(\mathcal{S}, 0)_{\infty}$ the space of arcs through 0 on $\mathcal{S}$. It can be canonically given the structure of a scheme over $\mathbb{C}$, as the projective limit of schemes of finite type obtained by truncating arcs at each finite order. So it makes sense to speak about its irreducible components $\left(C_{k}\right)_{k \in K}$. For each arc represented by an element in $C_{k}$, one can consider the intersection point with $E$ of its strict transform on $\widetilde{\mathcal{S}}_{m}$. For a generic element of $C_{k}$ (in the Zariski topology), this point is situated on a unique irreducible component of $E$. In this manner one defines a map

$$
\mathcal{N}:\left\{C_{k} \mid k \in K\right\} \longrightarrow\left\{E_{i} \mid i \in I\right\}
$$

which is called the Nash map associated to the germ $(\mathcal{S}, 0)$. It was defined by Nash around 1966, in a preprint published later as [17]. He proved that the map $\mathcal{N}$ is injective (which shows in particular that $K$ is a finite set) and asked the question:

\section{Is the map $\mathcal{N}$ bijective?}

This question is now called the Nash problem on arcs. No germ $(\mathcal{S}, 0)$ is known for which the answer is negative. But the bijectivity of $\mathcal{N}$ was only proved till now for special classes of singularities:

- for the germs of type $\left(\mathbb{A}_{n}\right)_{n \geq 1}$ by Nash himself in [17];

- for normal minimal singularities by Reguera [21]; different proofs were given by Plénat [19] and by Fernández-Sánchez [4];

- for sandwiched singularities it was sketched by Reguera [22], using her common work [15] with Lejeune-Jalabert on the wedge problem;

- for the germs of type $\left(\mathbb{D}_{n}\right)_{n \geq 4}$ by Plénat [19];

- for the germs with a good $\mathbb{C}^{*}$-action such that the curve Proj $\mathcal{S}$ is not rational, it follows immediately by combining results of Lejeune-Jalabert [13] and Reguera [22].

With the exception of the last class, all the other ones consist only in rational singularities and can be defined purely topologically.

Here we prove that the Nash map is bijective for a new class of surface singularities (Theorem 5.1), whose definition depends only on the intersection

TOME $134-2006-\mathrm{N}^{\mathrm{O}} 3$ 
matrix of the minimal resolution and not on the genera or possible singularities of the components $E_{i}$. In particular, their minimal resolution may not be good, which contrasts with the classes of singularities described before. The following (Corollary 5.2) is a particular case of the main theorem:

$$
\text { If } E \cdot E_{i}<0 \text { for any } i \in I \text {, then the Nash map } \mathcal{N} \text { is bijective. }
$$

We also show (Corollary 5.5) that the hypothesis of the previous corollary are verified by an infinity of topologically pairwise distinct non-rational singularities, which explains the title of the article.

The Nash map can also be defined in higher dimensions, over any field, for not necessarily normal schemes which admit resolutions of their singularities. It is always injective and the same question can also be asked. Ishii and Kollár proved in [6] (a good source for everything we use about spaces of arcs and the Nash map, as well as for references on related works) that it is not always bijective. Indeed, they gave a counterexample in dimension 4, which can be immediately transformed in a counterexample in any larger dimension. They left open the cases of dimensions 2 and $3 \ldots$

Acknowledgements. - We are grateful to the organizers of the Third Franco-Japanese Symposium and School on Singularities, Hokkaido University (Sapporo), September 13-17, 2004, during which the present collaboration was started. We are also grateful to M. Lejeune-Jalabert, M. Morales and the referee for their remarks.

\section{A criterion for distinguishing components of the space of arcs}

Consider a germ $(\mathcal{S}, 0)$ of normal surface and its minimal resolution morphism

$$
\pi_{m}:\left(\widetilde{\mathcal{S}}_{m}, E\right) \longrightarrow(\mathcal{S}, 0) .
$$

If $D$ is a divisor on $\widetilde{\mathcal{S}}_{m}$, it can be uniquely written as the sum of a divisor supported by $E$ - called the exceptional part of $D$ - and a divisor whose support meets $E$ in a finite number of points. If $D$ consists only of its exceptional part, we say that $D$ is purely exceptional.

For each $i \in I$, let $v_{E_{i}}$ be the divisorial valuation defined by $E_{i}$ on the fraction field of the analytic local $\operatorname{ring} \mathcal{O}_{\mathcal{S}, 0}$. Denote by $\mathfrak{m}_{\mathcal{S}, 0}$ the maximal ideal of this local ring. If $f \in \mathfrak{m}_{\mathcal{S}, 0}$, the exceptional part of $\operatorname{div}\left(f \circ \pi_{m}\right)$ is precisely

$$
\sum_{i \in I} v_{E_{i}}(f) E_{i} .
$$

For each component $E_{i}$ of $E$, consider the arcs on $\widetilde{\mathcal{S}}_{m}$ whose closed points are on $E_{i}-\bigcup_{j \neq i} E_{j}$ and which intersect $E_{i}$ transversally. Consider the set of 
their images in $(\mathcal{S}, 0)_{\infty}$ and denote its closure by $V\left(E_{i}\right)$. The sets $V\left(E_{i}\right)$ are irreducible and (see Lejeune-Jalabert [14, Appendix 3])

$$
(S, 0)_{\infty}=\bigcup_{i \in I} V\left(E_{i}\right)
$$

The following proposition is a special case of a general one proved by the first author in [18] (see also [19]) for non-necessarily normal germs of any dimension and for arbitrary resolutions. It generalizes an equivalent result proved by Reguera [21, Thm. 1.10] for the case of rational surface singularities.

Proposition 2.1. - If there exists a function $f \in \mathfrak{m}_{\mathcal{S}, 0}$ such that $v_{E_{i}}(f)<$ $v_{E_{j}}(f)$, then $V\left(E_{i}\right) \nsubseteq V\left(E_{j}\right)$.

Proof. - Let $(\mathcal{S}, 0) \hookrightarrow\left(\mathbb{C}^{n}, 0\right)$ be an analytic embedding of the germ $(\mathcal{S}, 0)$. Denote by $\left(x_{1}, \ldots, x_{n}\right)$ the coordinates of $\mathbb{C}^{n}$. An $\operatorname{arc} \phi \in(\mathcal{S}, 0)_{\infty}$ is then represented by $n$ formal power series

$$
\left(x_{k}(t)=\sum_{\ell=1}^{\infty} a_{k, \ell} t^{\ell}\right)_{1 \leq k \leq n},
$$

where the coefficients $\left(a_{k, \ell}\right)_{k, \ell}$ are subjected to algebraic constraints, coming from the fact that the arc must lie on $\mathcal{S}$.

For each $j \in I$, a Zariski open set $U_{f}\left(E_{j}\right)$ in $V\left(E_{j}\right)$ consists of the images by $\pi_{m}$ of the arcs on $\widetilde{\mathcal{S}}_{m}$ which meet transversely $E_{j}$ in a smooth point of $\operatorname{div}\left(f \circ \pi_{m}\right)$. If $\phi \in U_{f}\left(E_{j}\right)$, we have

$$
v_{E_{j}}(f)=v_{t}(f \circ \phi)
$$

where $v_{t}(g)$ denotes the order in $t$ of $g \in \mathbb{C}[[t]]$.

This shows that the first $v_{E_{j}}(f)-1$ coefficients of $f \circ \phi$, seen as elements of $\mathbb{C}\left[a_{k, \ell}\right]_{k, \ell}$, must vanish. Their vanishing defines a closed subscheme $Z_{f, j}$ of $(\mathcal{S}, 0)_{\infty}$. Therefore, $U_{f}\left(E_{j}\right) \subset Z_{f, j}$, which implies that

$$
V\left(E_{j}\right) \subset Z_{f, j}
$$

As $v_{E_{i}}(f)<v_{E_{j}}(f)$, we see that no element of $U_{f}\left(E_{i}\right)$ is included in $Z_{f, j}$, which shows that

The proposition follows.

$$
V\left(E_{i}\right) \nsubseteq Z_{f, j}
$$

\section{Construction of functions with prescribed divisor}

In this section, $\pi:(\widetilde{\mathcal{S}}, E) \rightarrow(\mathcal{S}, 0)$ denotes any resolution of $(\mathcal{S}, 0)$.

Inside the free abelian group generated by $\left(E_{i}\right)_{i \in I}$ we consider the set

$$
\mathcal{L}(\pi):=\left\{D \mid D \neq 0, D \cdot E_{i} \leq 0, \forall i \in I\right\} .
$$

TOME $134-2006-\mathrm{N}^{\mathrm{O}} 3$ 
It is a semigroup with respect to addition, which we call (following Lê $[11,3.2 .5]$ ) the Lipman semigroup associated to $\pi$ (see Lipman $[16, \S 18]$ ). It is known that it consists only of effective divisors (see Lipman $[16, \S 18$ (ii)]).

We call strict Lipman semigroup of $\pi$ the subset:

$$
\mathcal{L}^{0}(\pi):=\left\{D \in \mathcal{L}(\pi) \mid D \cdot E_{i}<0, \forall i \in I\right\}
$$

of the Lipman semigroup of $\pi$. It is always non-empty.

The importance of the Lipman semigroup comes from the fact that the exceptional parts of the divisors of the form $\operatorname{div}(f \circ \pi)$, where $f \in \mathfrak{m}_{\mathcal{S}, 0}$, are elements of it. The converse is true for rational surface singularities, but this is not the case for arbitrary surface singularities.

We give now a numerical criterion on a divisor $D \in \mathcal{L}(\pi)$ which allows one to conclude that it is the exceptional part of a divisor of the form $\operatorname{div}(f \circ \pi)$ :

Proposition 3.1. - Let D be an effective purely exceptional divisor, such that for any $i, j \in I$, one has the inequality:

$$
\left(D+E_{i}+K_{\widetilde{\mathcal{S}}}\right) \cdot E_{j}+2 \delta_{i}^{j} \leq 0
$$

where $\delta_{i}^{j}$ is Kronecker's symbol. Then there exists a function $f \in \mathfrak{m}_{\mathcal{S}, 0}$ such that the exceptional part of $\operatorname{div}(f \circ \pi)$ is precisely $D$.

Proof. - We use the following Grauert-Riemenschneider type vanishing theorem, proved by Laufer [9] for analytic germs and by Ramanujam [20] for algebraic ones (see also Bădescu $[1,4.1]$ ):

If $L$ is a divisor on $\widetilde{\mathcal{S}}$ such that $L \cdot E_{j} \geq K_{\widetilde{\mathcal{S}}} \cdot E_{j}$ for all $j \in I$, then

$$
H^{1}\left(\mathcal{O}_{\widetilde{\mathcal{S}}}(L)\right)=0 \text {. }
$$

We apply the theorem to $L=-D-E_{i}$, for any $i \in I$. Our hypothesis implies that $H^{1}\left(\mathcal{O}_{\widetilde{\mathcal{S}}}\left(-D-E_{i}\right)\right)=0$. Then, from the exact cohomology sequence associated to the short exact sequence of sheaves

$$
0 \rightarrow \mathcal{O}_{\widetilde{\mathcal{S}}}\left(-D-E_{i}\right) \longrightarrow \mathcal{O}_{\widetilde{\mathcal{S}}}(-D) \stackrel{\psi_{i}}{\longrightarrow} \mathcal{O}_{E_{i}}(-D) \rightarrow 0
$$

we deduce the surjectivity of the restriction map

$$
\psi_{i *}: H^{0}\left(\mathcal{O}_{\widetilde{\mathcal{S}}}(-D)\right) \longrightarrow H^{0}\left(\mathcal{O}_{E_{i}}(-D)\right) .
$$

By Serre duality on the irreducible (possibly singular) curve $E_{i}$ (see Reid $[23,4.10])$, we get

$$
h^{1}\left(\mathcal{O}_{E_{i}}(-D)\right)=h^{0}\left(\mathcal{O}_{E_{i}}\left(K_{\widetilde{\mathcal{S}}}+E_{i}+D\right)\right)=0 .
$$

For the last equality we have used the hypothesis $\left(D+E_{i}+K_{\widetilde{\mathcal{S}}}\right) \cdot E_{i} \leq-2<0$, which shows that the line bundle $\mathcal{O}_{E_{i}}\left(K_{\widetilde{\mathcal{S}}}+E_{i}+D\right)$ cannot have a non-trivial section. 
By applying the Riemann-Roch theorem and the adjunction formula for the irreducible curve $E_{i}$ of arithmetic genus $p_{a}\left(E_{i}\right)$ (see Reid $[23,4.11]$ ), we get

$$
\begin{aligned}
h^{0}\left(\mathcal{O}_{E_{i}}(-D)\right) & =h^{0}\left(\mathcal{O}_{E_{i}}(-D)\right)-h^{1}\left(\mathcal{O}_{E_{i}}(-D)\right)=\chi\left(\mathcal{O}_{E_{i}}(-D)\right) \\
& =1-p_{a}\left(E_{i}\right)-D \cdot E_{i} \\
& \geq 1-p_{a}\left(E_{i}\right)+\left(K_{\widetilde{\mathcal{S}}}+E_{i}\right) \cdot E_{i}+2 \\
& =1-p_{a}\left(E_{i}\right)+2 p_{a}\left(E_{i}\right)-2+2=1+p_{a}\left(E_{i}\right)>0 .
\end{aligned}
$$

This shows that there exists a non-identically zero section $s_{i} \in H^{0}\left(\mathcal{O}_{E_{i}}(-D)\right)$. The surjectivity of $\psi_{i *}$ implies that there exists $\sigma_{i} \in H^{0}\left(\mathcal{O}_{\widetilde{\mathcal{S}}}(-D)\right)$ such that $\psi_{i *}\left(\sigma_{i}\right)=s_{i}$. As $(\mathcal{S}, 0)$ is normal, there exists $f_{i} \in \mathfrak{m}_{\mathcal{S}, 0}$ with $\sigma_{i}=f_{i} \circ \pi$. If we write $D=\sum_{j \in I} a_{j} E_{j}$, we see that $v_{E_{i}}\left(f_{i}\right)=a_{i}$ and $v_{E_{j}}\left(f_{i}\right) \geq a_{j}$, for all $j \neq i$. We deduce that any generic linear combination $f=\sum_{i \in I} \lambda_{i} f_{i}$ of the functions so constructed verifies:

$$
v_{E_{i}}(f)=a_{i}, \quad \forall i \in I .
$$

The proposition is proved.

\section{REMARK 3.2}

a) The proof follows the same line as the one of Proposition 3.1 of [3] and 4.1 of [2], proved by Caubel, Némethi and Popescu-Pampu. The difference here is that we no longer deal with a $\operatorname{good}$ resolution of $(\mathcal{S}, 0)$ and we do not ask for a precise knowledge of the topological type of the total transform of $f$.

b) As Morales informed us, Laufer [10, 3.1] proved a related statement when $\pi$ is the minimal resolution of $(\mathcal{S}, 0)$ :

If $L$ is a line bundle on $\widetilde{\mathcal{S}}_{m}$ with $L \cdot E_{i} \geq 2 K_{\widetilde{\mathcal{S}}_{m}} \cdot E_{i}$, for all $i \in I$, then $L$ has no base points on $M$.

This implies immediately, by taking $L=\mathcal{O}_{\widetilde{\mathcal{S}}_{m}}(-D)$, that the conclusion of Proposition 3.1 is true with the hypothesis $\left(D+2 K_{\widetilde{\mathcal{S}}_{m}}\right) \cdot E_{i} \leq 0$, for all $i \in I$. As in the sequel we work only with the minimal resolution, we could have chosen to use Laufer's criterion. By looking at the way we use Proposition 3.1 in the proof of Proposition 4.3, one sees that this would have been enough in order to prove our main Theorem 5.1. Nevertheless, we think that Proposition 3.1 has independent interest. Indeed, neither Laufer's hypothesis nor ours implies the other one. In order to see it, consider first a singularity whose minimal resolution has an irreducible exceptional divisor $E$ which is smooth, of genus 2 and with $E^{2}=-1$ : then $D=4 E$ verifies our hypothesis, but not Laufer's. Secondly, by considering another singularity with $E=E_{1}+E_{2}, p_{a}\left(E_{1}\right)=$ $p_{a}\left(E_{2}\right)=0, E_{1}^{2}=-4, E_{2}^{2}=-2, E_{1} \cdot E_{2}=2$, one sees that $D=4 E_{1}+4 E_{2}$ verifies Laufer's hypothesis but not ours.

TOME $134-2006-\mathrm{N}^{\mathrm{O}} 3$ 


\section{The conditions $(*)$ and $(* *)$}

From now on, we deal again with the minimal resolution $\pi_{m}$ of the germ $(\mathcal{S}, 0)$.

Inside the real vector space with basis $\left(E_{i}\right)_{i \in I}$, we consider the open halfspaces

$$
\left\{\sum_{i \in I} a_{i} E_{i} \mid a_{i}<a_{j}\right\}
$$

for each $i \neq j$. We call them the fundamental half-spaces of $\pi_{m}$.

We introduce two conditions on the normal singularity $(\mathcal{S}, 0)$ :

$\left(^{*}\right)$ the intersection of $\mathcal{L}^{0}\left(\pi_{m}\right)$ with each fundamental half-space is non-empty;

(**) $E \in \mathcal{L}^{0}\left(\pi_{m}\right)$.

Notice that both conditions depend only on the intersection matrix of $E$. In particular, they are purely topological.

LEMMA 4.1. - The germs which verify condition $\left(^{* *}\right)$ form a strict subset of those which verify condition $(*)$.

Proof. — Let $(\mathcal{S}, 0)$ verify condition $\left.{ }^{* *}\right)$. Then, for $n \in \mathbb{N}^{*}$ big enough (in fact for $\left.n>\max _{i, j \in I}\left\{E_{i} \cdot E_{j} /\left|E \cdot E_{i}\right|\right\}\right)$, one sees from the definition of the strict Lipman semigroup that $n E+E_{j} \in \mathcal{L}^{0}\left(\pi_{m}\right)$, for all $j \in I$. But each fundamental half-space contains at least one of the divisors $n E+E_{j}$, which shows that $(\mathcal{S}, 0)$ verifies condition $(*)$.

Consider then any normal singularity $(\mathcal{S}, 0)$ whose minimal resolution has the same intersection matrix as a singularity of type $\mathbb{A}_{n}$ (that is, $E=\sum_{i=1}^{n} E_{i}$ with $E_{i}^{2}=-2$, for all $i \in\{1, \ldots, n\}, E_{i} \cdot E_{i+1}=1$, for all $i \in\{1, \ldots, n-1\}$ and $E_{i} \cdot E_{j}=0$, for all $i, j \in\{1, \ldots, n\}$ such that $\left.|i-j| \notin\{0,1\}\right)$, the components $E_{i}$ having otherwise arbitrary genera and singularities. Take $n \geq 3$. Then $E \cdot E_{i}=0$, for all $i \in\{2, \ldots, n-1\}$, which shows that $E \notin \mathcal{L}^{0}\left(\pi_{m}\right)$. That is, the germ $(\mathcal{S}, 0)$ does not verify condition $(* *)$.

Define

$$
\alpha_{k}:=n k-\frac{(k-1) k}{2}
$$

for any $k \in\{1, \ldots, n\}$. Then it is immediate to see from the definitions that the divisors $D:=\sum_{k=1}^{n} \alpha_{k} E_{k}$ and $D^{\prime}:=\sum_{k=1}^{n} \alpha_{n+1-k} E_{k}$ are in the strict Lipman semigroup and that each fundamental half-space contains exactly one of them. Therefore, the germ $(\mathcal{S}, 0)$ verifies condition $\left(^{*}\right)$. This shows that the inclusion stated in the lemma is strict.

\section{REMARK 4.2}

a) We choose to distinguish inside the class of singularities which verify condition $(*)$ those which verify condition $(* *)$ for computational convenience, 
because this second condition is more readily verified on a given intersection matrix.

b) $\mathbb{A}_{n}$-type singularities are the only rational double points which verify condition $(*)$. This illustrates the difficulty of dealing with $\mathbb{D}_{n}$-type singularities (see Plénat [19]).

The motivation to introduce condition $(*)$ comes from the following proposition:

Proposition 4.3. - Suppose that $(\mathcal{S}, 0)$ verifies condition $(*)$. Then, for any pair of distinct indices $i, j \in I$, there exists a function $f \in \mathfrak{m}_{\mathcal{S}, 0}$ such that

$$
v_{E_{i}}(f)<v_{E_{j}}(f) .
$$

Proof. - Let $i, j \in I$ satisfy $i \neq j$. As $(\mathcal{S}, 0)$ verifies condition $(*)$, there exists $D=\sum_{\ell \in I} a_{\ell} E_{\ell} \in \mathcal{L}^{0}\left(\pi_{m}\right)$ such that $a_{i}<a_{j}$. Then, for $n \in \mathbb{N}^{*}$ big enough, one has $\left(n D+E_{k}+K_{\widetilde{\mathcal{S}}}\right) \cdot E_{\ell}+2 \delta_{k}^{\ell} \leq 0$, for all $k, \ell \in I$. By Proposition 3.1, there exists $f \in \mathfrak{m}_{\mathcal{S}, 0}$ such that $\operatorname{div}\left(f \circ \pi_{m}\right)=n D$, which shows that $v_{E_{i}}(f)=$ $n a_{i}<n a_{j}=v_{E_{j}}(f)$.

REMARK 4.4. - The referee suggests us the following alternative proof of the previous proposition. Let $D=\sum_{\ell \in I} a_{\ell} E_{\ell} \in \mathcal{L}^{0}\left(\pi_{m}\right)$ such that $a_{i}<a_{j}$. Then $\mathcal{O}_{\widetilde{\mathcal{S}}_{m}}(-D)$ is ample (see Lipman [16, 10.4 and proof of 12.1 (iii)]). Thus there exists $n \in \mathbb{N}^{*}$ such that $\mathcal{O}_{\widetilde{\mathcal{S}}_{m}}(-n D)$ is generated by its global sections. Therefore there exists $f \in \mathfrak{m}_{\mathcal{S}, 0}$ such that the exceptional part of $\operatorname{div}(f \circ \pi)$ is precisely $n D$. For such an $f$, we have of course $v_{E_{i}}(f)<v_{E_{j}}(f)$.

\section{The proof of the main theorem}

Our main theorem is:

THEOREM 5.1. - Suppose that $(\mathcal{S}, 0)$ verifies condition $\left(^{*}\right)$. Then the Nash map $\mathcal{N}$ is bijective.

Proof. - By combining Proposition 2.1 and Proposition 4.3, we deduce that $V\left(E_{i}\right) \nsubseteq V\left(E_{j}\right)$ for any $i \neq j$. As $(\mathcal{S}, 0)_{\infty}=\bigcup_{i \in I} V\left(E_{i}\right)$, we deduce that the schemes $\left(V\left(E_{i}\right)\right)_{i \in I}$ are precisely the irreducible components $\left(C_{k}\right)_{k \in K}$ of $(\mathcal{S}, 0)_{\infty}$. As the Nash map $\mathcal{N}$ is injective, this shows its surjectivity. The theorem is proved.

Using Lemma 4.1, we get as an immediate corollary:

Corollary 5.2. - Suppose that $(\mathcal{S}, 0)$ verifies condition $\left({ }^{* *}\right)$. Then the Nash map $\mathcal{N}$ is bijective.

TOME $134-2006-\mathrm{N}^{\mathrm{O}} 3$ 
We denote by $\Gamma(E)$ the dual graph of $E$, whose vertices correspond bijectively to the components $\left(E_{i}\right)_{i \in I}$, the vertex $E_{i}$ being weighted by $E_{i}^{2}$ and the vertices $E_{i}, E_{j}$ being joined by $E_{i} \cdot E_{j}$ vertices, for any $i \neq j$. Let $\gamma\left(E_{i}\right)$ denote the number of edges which start from the vertex $E_{i}$ (so, each loop based at the vertex $E_{i}$ counts for 2).

The next proposition characterizes rational singularities among those which verify condition $(* *)$.

Proposition 5.3. - A singularity which verifies condition $\left({ }^{* *}\right)$ is rational if and only if the following conditions are simultaneously verified:

(i) $\Gamma(E)$ is a tree;

(ii) $E_{i} \simeq \mathbb{P}^{1}$, for all $i \in I$;

(iii) $\left|E_{i}^{2}\right|>\gamma\left(E_{i}\right)$, for all $i \in I$.

Proof. - First of all, notice that conditions (i) and (ii) imply that $\pi_{m}$ is a good resolution.

Suppose that $(\mathcal{S}, 0)$ is rational and verifies condition $\left({ }^{* *}\right)$. Then conditions (i) and (ii) are verified, as general properties of rational singularities (see Bădescu $[1,3.32 .3])$. As $E \cdot E_{i}=-\left|E_{i}^{2}\right|+\gamma\left(E_{i}\right)$, condition (iii) is also verified.

Conversely, suppose that the conditions (i), (ii) and (iii) are verified. By a result of Spivakovsky [24, II] (see also Lê $[12,5.3]$ ), this shows that $(\mathcal{S}, 0)$ is a normal minimal singularity, and in particular it is rational. Consult the references above for the notion of minimal surface singularity, as well as Kollár [7], where this notion was introduced in arbitrary dimensions.

REMARK 5.4

a) Normal minimal surface singularities are precisely those whose minimal good resolution verifies conditions (i), (ii) and

(iii) $)^{\prime}:\left|E_{i}^{2}\right| \geq \gamma\left(E_{i}\right)$, for all $i \in I$.

The stronger condition (iii) is equivalent to the fact that the minimal resolution can be obtained by blowing-up once the origin (see Spivakovsky [24, II], Lê $[12,6.1])$ : one says that the singularity is superisolated. Thus, Proposition 5.3 is equivalent to the fact that the rational surface singularities which verify condition $(* *)$ are precisely the superisolated minimal ones (a remark we owe to Lejeune-Jalabert).

b) There are rational singularities which verify condition $(*)$ but do not verify condition $(* *)$. Consider for example a germ of normal surface whose minimal resolution $\pi_{m}$ is good and has a reduced exceptional divisor $E$ with four components of genus 0 such that

$$
\begin{gathered}
E_{1}^{2}=E_{2}^{2}=E_{3}^{2}=-n \leq-5, \quad E_{4}^{2}=-2, \\
E_{1} E_{4}=E_{2} E_{4}=E_{3} E_{4}=1, \quad E_{1} E_{2}=E_{2} E_{3}=E_{3} E_{1}=0 .
\end{gathered}
$$

BULletin DE LA SOCIÉtÉ MATHÉMATIQUE DE FRANCE 
Then it is immediate to verify that the divisors

$$
\begin{gathered}
(2 n+1)\left(E_{1}+E_{2}+E_{3}\right)+4 n E_{4}, \\
\left(2 n^{2}-2 n+3\right) E_{1}+3 n\left(E_{2}+E_{3}\right)+\left(n^{2}+3 n\right) E_{4}
\end{gathered}
$$

as well as those obtained by permuting $E_{1}, E_{2}, E_{3}$ are in $\mathcal{L}^{0}\left(\pi_{m}\right)$ and that each fundamental half-space contains at least one of them. So, the germ verifies condition $(*)$. But, as $E \cdot E_{4}>0$, the germ does not verify condition (**). Moreover, the dual graph $\Gamma(E)$ is a subgraph of the dual graph associated to the resolution of a plane curve singularity (attach $n-1$ vertices to $E_{1}$, $n-2$ vertices to $E_{2}$ and $n-3$ vertices to $E_{3}$, all of them weighted by -1 ). Using Lê $[12,4.8]$ (see also Spivakovsky [24, II]), we see that the singularity is sandwiched, and in particular it is rational.

As condition (iii) in the Proposition 5.3 is equivalent with condition (**), we see immediately that any (abstract) graph is the dual graph of the minimal resolution of a singularity which verifies condition $(* *)$, once the weights of the vertices are negative enough. Then, if one of the conditions (i) or (ii) is not satisfied, we are in presence of a non-rational singularity. This shows:

COROLlary 5.5. - There exists an infinity of pairwise topologically distinct normal non-rational surface singularities which verify condition $\left(^{* *}\right)$, and consequently for which the Nash map is bijective.

\section{BIBLIOGRAPHY}

[1] Bădescu (L.) - Algebraic Surfaces, Springer, 2001.

[2] Caubel (C.), Némethi (A.) \& Popescu-Pampu (P.) - Milnor open books and Milnor fillable contact 3-manifolds, Topology, t. 45 (2006), pp. 673-689.

[3] Caubel (C.) \& Popescu-Pampu (P.) - On the contact boundaries of normal surface singularities, C. R. Acad. Sci. Paris, Sér. I, t. 339 (2004), pp. 43-48.

[4] Fernández-SÁnChez (J.) - Equivalence of the Nash conjecture for primitive and sandwiched singularities, Proc. Amer. Math. Soc., t. 133 (2005), pp. 677-679.

[5] Grauert (H.) - Über Modifikationen und exzeptionnelle analytische Mengen, Math. Ann., t. 146 (1962), pp. 331-368.

[6] Ishit (S.) \& Kollár (J.) - The Nash problem on arc families of singularities, Duke Math. J., t. 120 (2003), pp. 601-620.

[7] Kollár (J.) - Toward moduli of singular varieties, Comp. Math., t. 56 (1985), pp. 369-398.

TOME $134-2006-\mathrm{N}^{\mathrm{O}} 3$ 
[8] Laufer (H.) - Normal two-dimensional Singularities, Princeton Univ. Press, 1971.

[9] _ On rational singularities, Amer. J. Math., t. 94 (1972), pp. 597608.

[10] Weak simultaneous resolution for deformations of Gorenstein surface singularities, Part 2, Proc. Symp. Pure Math., t. 40 (1983), pp. 1-29.

[11] LÊ (D. T.) - Geometry of complex surface singularities. Singularities, Sapporo 1998, in Advanced Studies in Pure Math., vol. 29, 2000, pp. 163180.

[12] Les singularités Sandwich. Resolution of Singularities. A research textbook in tribute to Oscar Zariski. Based on the courses given at the Working Week in Obergurgl, Austria, September 7-14, 1997, in Progress in Math., vol. 181, 2000, pp. 457-483.

[13] Lejeune-Jalabert (M.) - Arcs analytiques et résolution minimale des singularités des surfaces quasi-homogènes. Séminaire sur les singularités des surfaces, Palaiseau, 1976-1977, M. Demazure, H. Pinkham, B. Teissier eds., in Lecture Notes in Math., vol. 777, Springer, 1980.

[14] Courbes tracées sur un germe d'hypersurface, Amer. J. Math., t. 112 (1990), pp. 525-568.

[15] Lejeune-Jalabert (M.) \& Reguera (A.) - Arcs and wedges on sandwiched surface singularities, Amer. J. Math., t. 121 (1999), pp. 1191-1213.

[16] LiPMAN (J.) - Rational singularities with applications to algebraic surfaces and unique factorization, Publ. Math. Inst. Hautes Études Sci., t. 36 (1969), pp. 195-279.

[17] Nash (J. F.) - Arc structure of singularities, Duke Math. J., t. 81 (1995), pp. 31-38.

[18] Plénat (C.) - A propos du problème des arcs de Nash, Ann. Inst. Fourier, t. 55 (2005), pp. 805-823.

[19] L Résolution du problème des arcs de Nash pour les points doubles rationnels $D_{n},(n \geq 4)$, Thèse, Univ. Paul Sabatier, Toulouse (Septembre 2004), available at http://fermat.ups-tlse.fr/\%7Ewebthesards/ theses.htm.

[20] Ramanujam (C. P.) - Remarks on the Kodaira vanishing theorem, J. Indian Math. Soc., t. 36 (1972), pp. 41-51.

[21] Reguera (A. J.) - Families of arcs on rational surface singularities, Manuscripta Math., t. 88 (1995), pp. 321-333.

[22] - Image of the Nash map in terms of wedges, C. R. Acad. Sci. Paris, Sér. I, t. 338 (2004), pp. 385-390.

[23] ReId (M.) - Chapters on Algebraic Surfaces, in Complex Algebraic Geometry. J. Kollár ed., Amer. Math. Soc., 1997, pp. 3-159. 
[24] SpIvakovsky (M.) - Sandwiched singularities and desingularization of surfaces by normalized Nash transformations, Annals of Math., t. 131 (1990), pp. 411-491. 